\title{
The DP5 Probability, Quantification and Visualisation of Structural Uncertainty in Single Molecules
}

\author{
Alexander Howarth, ${ }^{\text {a Jonathan M. Goodman*a }}$ \\ Whenever a new molecule is made, a chemist will justify the proposed structure by analysing the NMR spectra. The widely- \\ used DP4 algorithm will choose the best match from a series of possibilities, but draws no conclusions from a single candidate \\ structure. Here we present the DP5 probability, a step-change in the quantification of molecular uncertainty: given one \\ structure and one ${ }^{13} \mathrm{C}$ NMR spectra, DP5 gives the probability of the structure being correct. We show the DP5 probability \\ can rapidly differentiate between structure proposals indistinguishable by NMR to an expert chemist. We also show in a \\ number of challenging examples the DP5 probability may prevent incorrect structures being published and later reassigned. \\ DP5 will prove extremely valuable in fields such as discovery-driven automated chemical synthesis and drug development. \\ Alongside the DP4-AI package, DP5 can help guide synthetic chemists when resolving the most subtle structural uncertainty. \\ The DP5 system is available at https://github.com/Goodman-lab/DP5.
}

\section{Introduction}

Molecular structure elucidation and verification are central problems in organic, synthetic and natural product chemistry. Due to richness of the structural information its spectra contain, NMR spectroscopy has cemented itself as the method chemists use to solve these problems. Due to the complex nature of NMR spectra and often subtle variation between similar molecules, interpretation of these spectra can sometimes present a significant challenge. As a result, incorrectly assigned structures remain pervasive in the literature. ${ }^{1}$ Many of these incorrectly assigned are only discovered after costly and time consuming total syntheses are completed revealing a discrepancy between the experimental and literature NMR data. ${ }^{2,34}$

Over the last two decades, many computational tools have been developed to aid the assignment of NMR spectra and elucidation of molecular structures. ${ }^{5-7}$ Comparing experimental NMR shifts with those calculated for a candidate structure using density functional theory (DFT) is now a well-established methodology and has been used to solve the structures of many molecules. ${ }^{8-11}$ A powerful way of performing this analysis is to calculate the DP4 probability. ${ }^{12}$ Unlike comparative metrics such as MAE and CMAE, the DP4 algorithm applies Bayes Theorem to calculate the probability that each candidate structure is the correct one. DP4 requires a list of possible structures as its input, and it assumes that one of these structures is correct. It is common for structures to be determined except for uncertainty in the details of their stereochemistry. DP4 has proved invaluable in the resolution of many such cases. ${ }^{13-17}$ DP4 can also be used to resolve nonstereochemical uncertainty, provided that all of the acceptable possible structures can be enumerated. However, in cases where all the proposed candidate structures may be incorrect or only a single structure has been proposed, DP4 analysis cannot be applied. Until now in these very common situations chemists would have no quantitative way of assessing the probability of their proposed structure being correct given the NMR spectra.

To solve this problem, we present the DP5 probability, a new methodology and complete software package for quantifying uncertainty in molecular structures. Similar to the DP4 probability, the DP5 probability gives the probability that a candidate structure is correct. However, in contrast to DP4, DP5 calculates normalised stand-alone probabilities and hence, the user can propose one or many structures without having to assume any of their proposals are correct. As a result, DP5 can be used to answer different questions to DP4 and will prove valuable in situations where this type of analysis was previously impossible. The DP5 probability is calculated given only the onedimensional ${ }^{13} \mathrm{C}$ NMR data and utilises the same computational engine as the latest iteration of our DP4 software, DP4-AI. This program manages all NMR processing, assignment, DFT calculations and statistical modelling automatically. DP5 can also be used on a case-by-case basis utilising the graphical user interface (GUI).

The system was developed and rigorously tested utilising a dataset of 5140 organic molecules from NMRShiftDB ${ }^{18}$. To demonstrate the performance of the DP5 probability in even

\footnotetext{
a.A Centre for Molecular Informatics, Yusuf Hamied Department of Chemistry, University of Cambridge, Lensfield Road, Cambridge, CB2 1EW. Electronic Supplementary Information (ESI) available: The supporting information contains a detailed description of the program, the full results from the program evaluation all assigned spectra and NMR shift calculations. Results of all DFT and MM calculations are available from https://doi.org/10.17863/CAM.65145 See DOI: $10.1039 / \mathrm{x} 0 \times x \times 0000 x$
} 


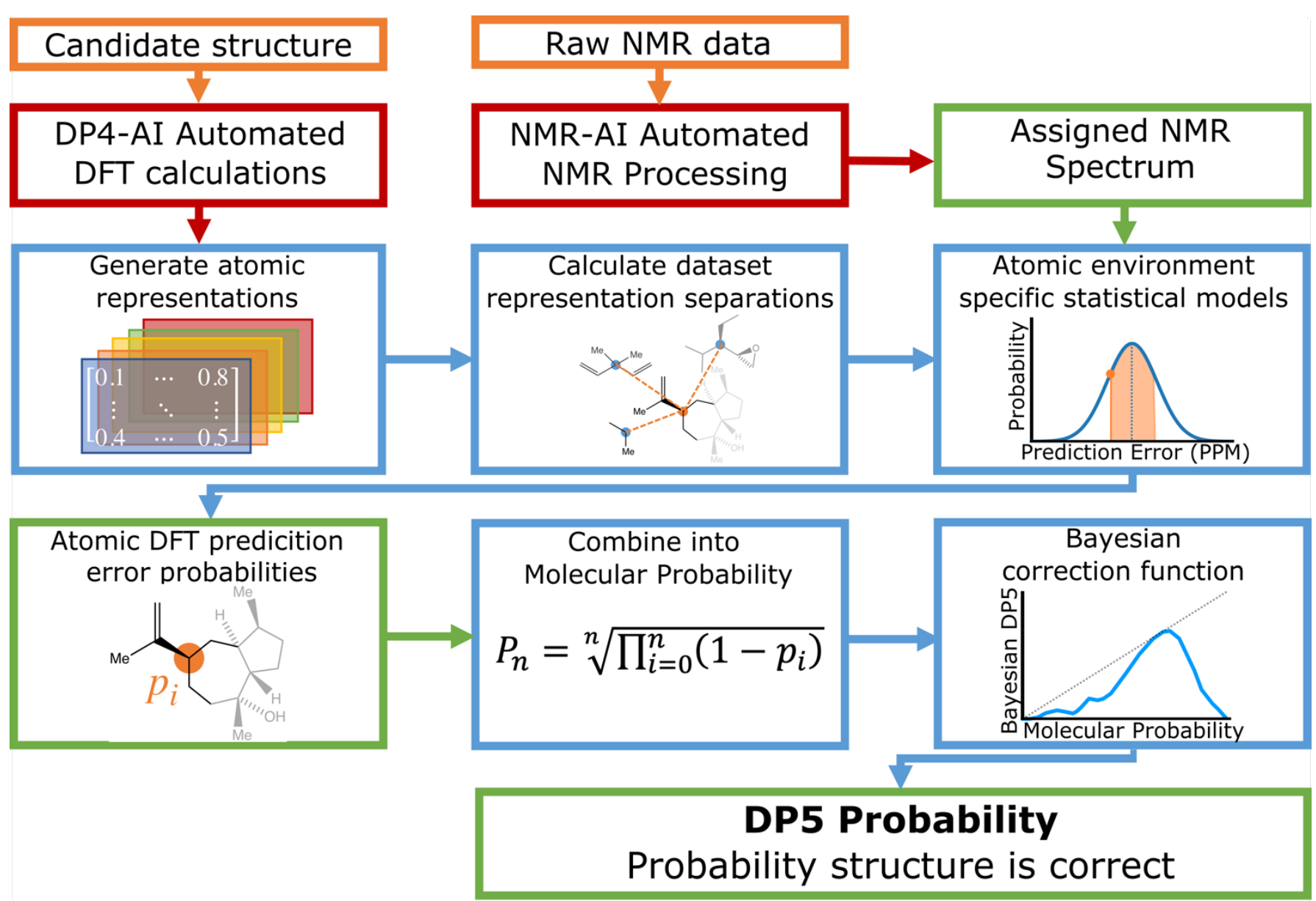

Figure 1. Schematic of the DP5 program. The required inputs from the user are a candidate structure and the raw ${ }^{13} \mathrm{C} \mathrm{NMR} \mathrm{data} \mathrm{(or} \mathrm{a}$ list NMR signals). The DP5 probability is built on top of the DP4-Al analysis.

more challenging situations, the system was also evaluated on undergone reassignments in the literature.

DP5 represents an exciting leap forward in quantifying molecular uncertainty. This system will prove valuable in fields requiring high throughput molecular structure elucidation such as automated chemical synthesis, but also in traditional organic chemistry as a tool to aid and guide expert chemists in their development of complex syntheses. It has been made possible following recent advances in molecular machine learning techniques and increased data availability. ${ }^{19-24}$

\section{Computational Methods}

DFT calculations for the structure reassignment examples were performed using the method developed in previous works. ${ }^{25-27}$ All molecular mechanics calculations were performed using MacroModel (Version 9.9) ${ }^{39}$. All conformational searches were performed in the gas phase utilizing the MMFF force field ${ }^{40-45}$ and a mixture of Low Mode following and Monte Carlo search algorithms. ${ }^{46,47}$ The step count for MacroModel was set so that all low energy conformers were found at least 5 times. Quantum mechanical calculations were carried out using Gaussian0948. NMR shielding constants were found using the GIAO method. ${ }^{49-51}$ The functional mPW1PW9152 was chosen with the $6-311 G(d)^{53,54}$ basis set for NMR shift prediction as this has been shown to be optimal for DP4 calculation. For molecules containing iodine, the basis set def2-SVP55,56 was chosen. All DFT calculations were performed using the implicit PCM solvent model. ${ }^{57}$ The molecular geometries were also optimized at the DFT level of theory, this was performed using the B3LYP functional58,59 with the $6-31 \mathrm{G}(\mathrm{d})$ basis set. Finally, single-point energies were separately calculated using M06$2 X^{60}$ functional and def2-TZVP55,56 basis set.

The calculations were managed by the DP4-Al27 Python script written in Python 3.7. DP4-Al is available from http://wwwjmg.ch.cam.ac.uk/tools/nmr/ and GitHub https://github.com/orgs/Goodman-lab/.

DFT optimised geometries and NMR shift calculations for the molecules from NMRShiftDB were obtained from the training data of the GNN NMR shift prediction software CASCADE. ${ }^{61} \mathrm{~A}$ single conformer of each of these molecules was optimised utilising the M062X functional and def2-TZVP basis set and NMR shift calculations performed using in $6-311 \mathrm{~g}(\mathrm{~d})$ basis set and mPW1PW91 functional.

Calculation of FCHL atomic representations, 12 distances and gaussian kernel transformations were performed using the python package qml. ${ }^{62}$

\section{Program Description}


The main text of the article should appear here with headings as appropriate. A schematic of the DP5 program is displayed in Figure 1 . The required input files are the structure of the molecule and either raw ${ }^{13} \mathrm{C}$ NMR data or a list of NMR peaks.

DP5 calculates NMR shifts for the atoms in populated conformers of the candidate structure utilising the highly optimised and well established methods within DP4-Al (see supporting information section 2.1)..$^{25-27}$

NMR interpretation is handled by a part of DP4-Al called NMRAl. ${ }^{27}$ This system was developed to remove the requirement for the user to process and assign NMR spectra and has been demonstrated to complete this task to at least the same high standard as an expert chemist.

Once the geometries of populated conformers have been calculated, the probabilities of the observed DFT-NMR prediction errors for each atom in that conformer can be found. To do this a probability density function (PDF) describing the DFT-NMR prediction error distribution is required (see supporting information section S2.2). This PDF is found empirically by performing a Kernel density estimation (KDE) on a dataset of 63542 known prediction errors calculated for the DFT optimised geometries of 5140 molecules from NMRShiftDB. It is well known that the expected magnitude and variance of DFT prediction errors for different functionals show strong complex, nonlinear dependencies on atomic environment. 28,29 This process is made to take into account the properties of the specific atomic environment by weighting each training point by its similarity to the test point. This similarity is calculated by finding the Euclidian distance between a vector representation of the test atomic environment and those in the training set. These distances are converted into covariances utilising a gaussian kernel (equation (1); see supporting information section S2.4). By setting the pre-exponential scaling factor to one, these covariances can be interpreted as a measure of the similarity. The resulting PDF is integrated by equation (2) (see supporting information section S2.5) to yield a prediction error probability for each atom in the conformer. Once atomic probabilities have been calculated for each conformer, these values are Boltzmann weighted to produce overall atomic

$$
\operatorname{similarity~}_{i j}=\mathrm{A} \exp \left(-\frac{\left\|X_{i}-X_{j}\right\|_{2}^{2}}{2 \sigma^{2}}\right)
$$

The similarity between the $\mathrm{FCHL}$ representation of atom $i, X_{i}$ and that of atom $j$ in the test set $X_{j}$ is calculated using a gaussian kernel.

$$
\mathrm{p}_{i}=\int_{-\left|\bar{\Delta}-\Delta_{i}\right|}^{+\left|\bar{\Delta}-\Delta_{i}\right|} p d f_{i}(\Delta) d \Delta
$$

A prediction error probability for atom $i$ is calculated by integrating the bespoke prediction error function generated for that atom, where $\Delta_{i}$ is the (internally scaled) prediction error for atom $i$ and $\bar{\Delta}$ corresponds to the mean absolute prediction error for the training set.

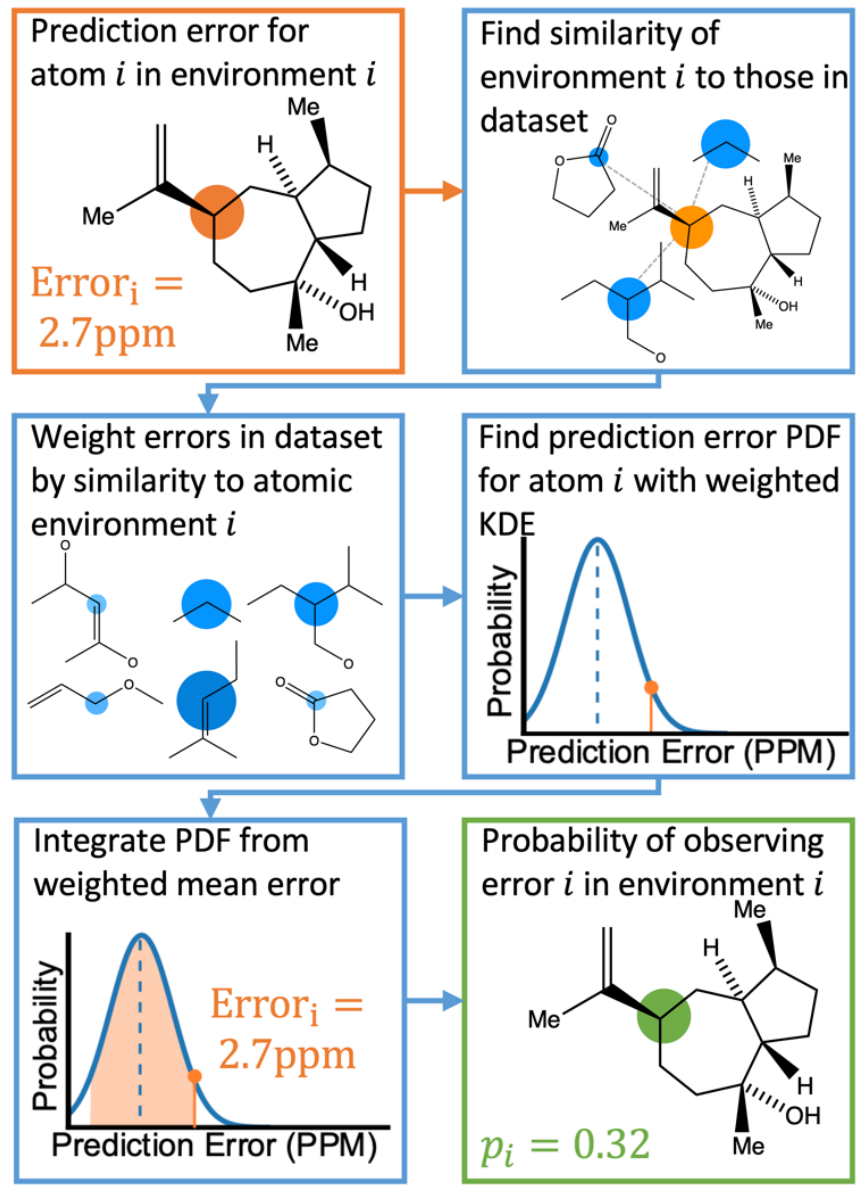

Figure 2. Schematic diagram of how the probability of observing a DFT-NMR prediction error for an atom in a given environment is calculated as described in the text.

probabilities for the structure. This process is summarised in Figure 2 .

The representation used by DP5 was investigated in great detail. In recent years many molecular representations have been developed for applications in molecular machine learning, such as the coulomb matrix, ${ }^{30}$ bag-of-bonds, ${ }^{31}$ aSLATM $^{32}$ and FCHL. ${ }^{23}$ Kernel ridge regression (KRR) utilising the $\mathrm{FCHL}$ atomic representation have been shown to predict NMR shielding constants with near chemical accuracy (also tested in this work see supporting information section S3.4.1).33 These works demonstrate that $\mathrm{FCHL}$ contains the information required to accurately encode atomic environments. Due to the similarity of these tasks, the FCHL representation has been chosen for use in the DP5 probability calculation (see supporting information section S2.3).

A particular challenge in the development of the DP5 probability was determining an equation to combine individual atomic probabilities to yield probability for the whole structure. If any single atom is given too much influence, a molecular probability of one or zero will usually be assigned, whilst if there is too much smoothing over individual atomic probabilities, the resulting molecular probabilities will not show enough useful variation. A number of formulae were tested during this study (see supporting information section S2.7). Overall equation (3) was found to yield useful variation in molecular probabilities, 
whilst combining the atomic probabilities in a mathematically meaningful way. The inclusion of the geometric mean in equation (3) was found to be necessary to prevent single atoms with a very high or low probability having too much influence on the final result.

The last stage in the calculation scales the molecular probability using a Bayesian correction function to yield the final DP5 probability (see supporting information section S2.8). This empirical stage of the process ensures the DP5 probability assigned matches the probability of the structure being correct as closely as possible. This empirical correction function was found by first calculating a PDF for the molecular probabilities of the 5140 NMRShiftDB molecules. By finding all the possible pairs of spectra and structures in this dataset with the same number of carbon atoms, a PDF of the molecular probabilities of incorrect spectra-structure pairs was also generated. In this instance, each pair was assigned a weight to ensure the mean absolute DFT-NMR prediction error distribution of these incorrect pairs matched that of the correct structure-spectra pairs (see supporting information section S3.2). Given any proposed structure must be either correct or incorrect, by applying Bayes Theorem the DP5 probability is defined by equation (4).

$$
\mathrm{P}_{\mathrm{n}}=\sqrt[n]{\left(\prod_{i=0}^{n} 1-p_{i}\right)}
$$

Atomic DP5 probabilities are combined to form the molecular probability $P_{n}$ by equation 3 , where $n$ is the number of atoms in the molecule and $p_{i}$ is the DFTNMR prediction error probability for atom $i$

$$
\mathrm{DP} 5=\frac{\mathrm{P}\left(\text { correct } \mid \mathrm{P}_{\mathrm{n}}\right)}{\mathrm{P}\left(\text { incorrect } \mid \mathrm{P}_{\mathrm{n}}\right)+\mathrm{P}\left(\text { correct } \mid \mathrm{P}_{\mathrm{n}}\right)}
$$

The DP5 probability is calculated by applying Bayes Theorem to the molecular probability calculated in equation 3 , where $\mathrm{P}\left(\right.$ correct $\left.\mid \mathrm{P}_{\mathrm{n}}\right)$ gives the probability of a molecule being correct given its calculated molecular probability $\mathrm{P}_{\mathrm{n}}$.

Calculation of the DP5 probability has been integrated into the well-established DP4-AI workflow. ${ }^{27}$ All the required calculations and analysis of NMR data can be performed automatically with no user input required. DP5 can hence be integrated into pre-existing automatic reaction/characterisation workflows. DP5 analysis can also be performed on single molecules with the GUI. This GUI can be used to launch calculations, analyse NMR assignments made by NMR-Al and also to investigate the DP5 statistics. The GUI visually displays the atomic probabilities, helping the chemist identify potential regions of the molecule that may be incorrect and determine possible modifications (Figure 3 ).

\section{Results}

\section{Atomic DP5 Probabilities Quantitatively Highlight Regions of the Molecule Likely to be Incorrect}

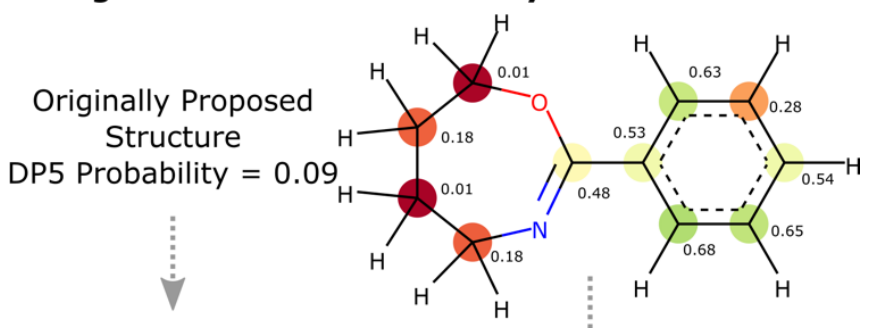

Revise regions with high DP5 probabilities

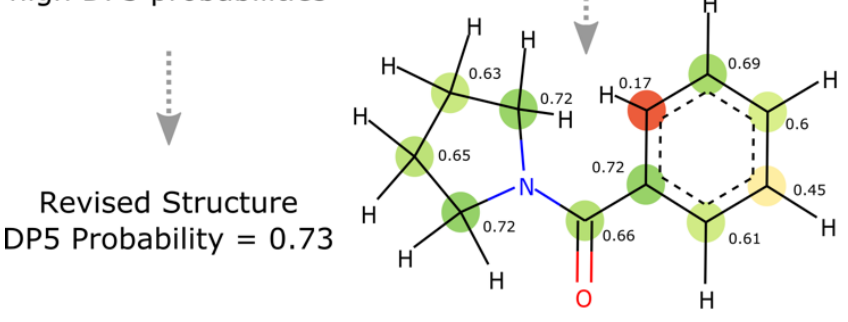

Probability of Atom Being Correct

$0.0 \square 1.0$

Figure 3. The GUI accompanying DP5 pictorially overlays atomic DP5 probabilities onto the molecular structure. This clearly displays regions of the structure that are expected to be correct and conversely regions that may require revision. This functionality will help chemistry assess and revise structure proposals. This structure revision example has been taken from a real-world case study of an incorrectly assigned molecule in the literature (see Results)

The main text of the article should appear here with headings as appropriate. A major challenge in the development of DP5 involved constructing a method to assess the efficacy of the system. As the DP5 probability is not a physical property that can be measured, it is not straightforward to compare the DP5 probability assigned to a molecule with an experimental value. In this study two rigorous evaluation methods were devised to assess and improve the real-world effectiveness of the DP5 probability.

The database of 5140 organic molecules from NMRShiftDB as used in comprehensive leave-one-out style cross validation study summarised in Figure 4 (see SI 3.2). In this study DP5 analysis of correct and incorrect proposed candidate structures was simulated by permuting the experimental data to form correct and incorrect pairs. This analysis is particularly powerful as negative examples could be synthesised from real world data, avoiding more unreliable methods involving generating fake experimental or calculated spectra. This analysis was used to develop DP5 and was repeated for many different formulations of the DP5 probability (see supporting information section S4.1). The results for the final DP5 system can be seen in Figure 4. The final DP5 methodology was also evaluated against a series of thirteen real world structure reassignment problems from the literature, molecules S1a-S13b in Figure 5. This study represents a very significant challenge for DP5 and will evaluate its performance in a regime where even the most 

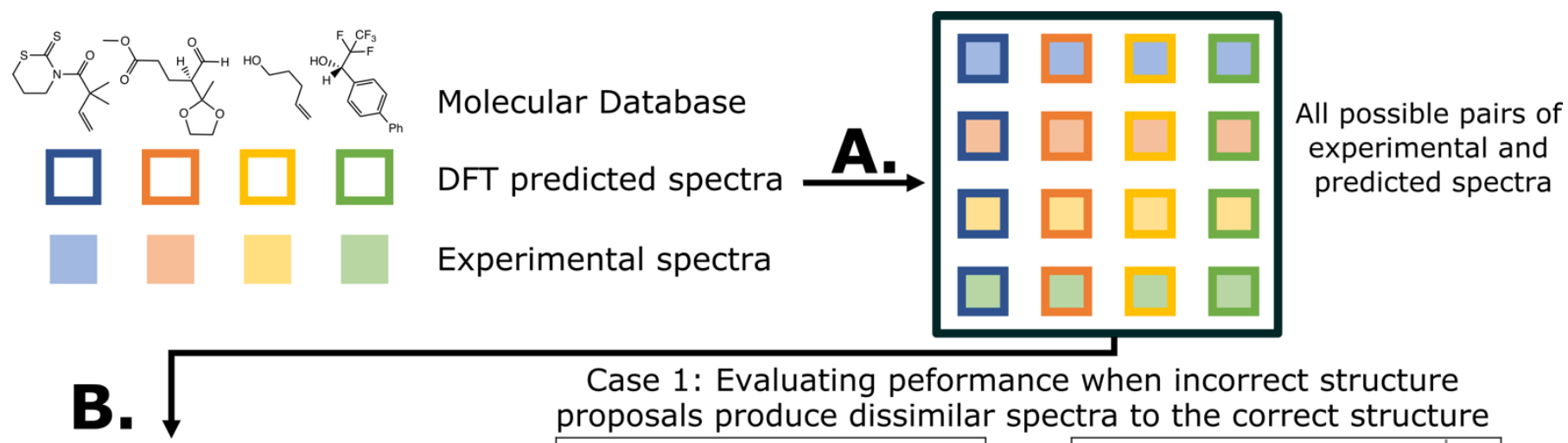

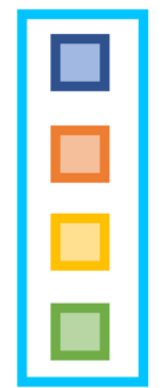

Correct pairs
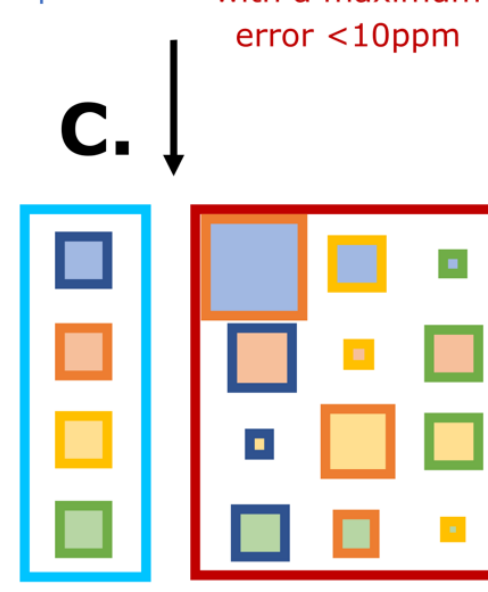

Correct pairs

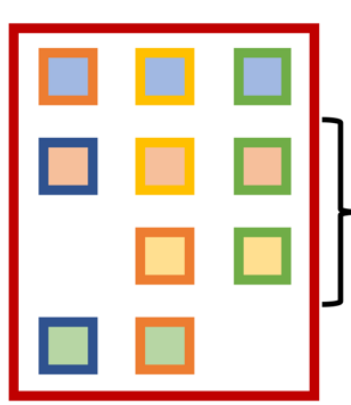

All incorrect pairs with a maximum error <10ppm

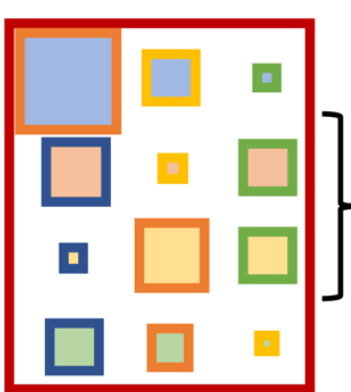

Incorrect pairs weighted by the probability of a correct pair having the same MAE
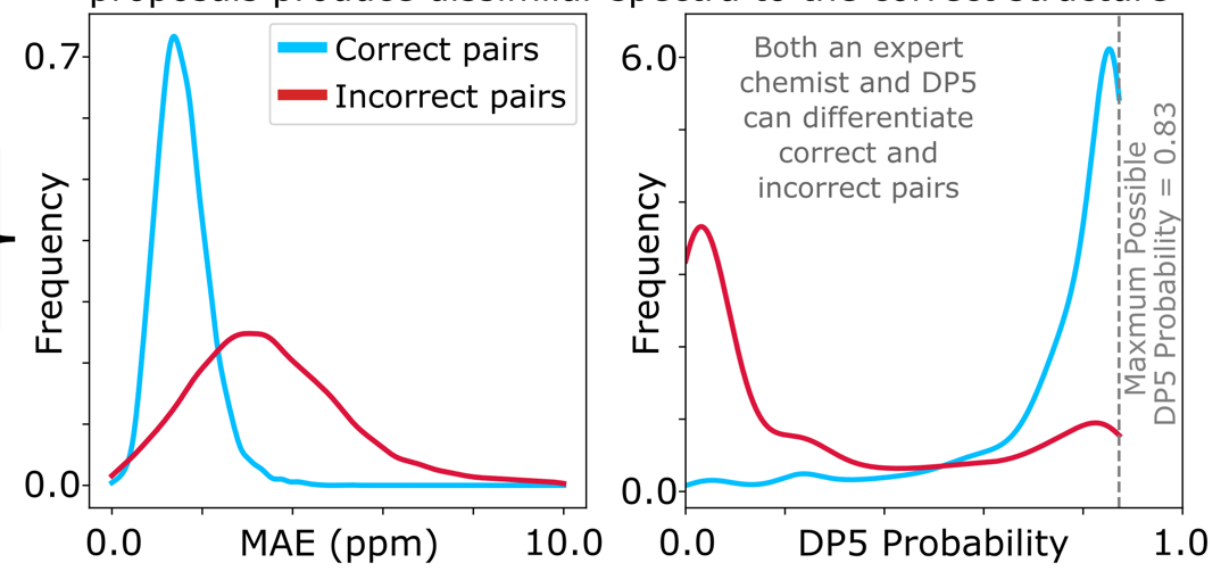

Case 2: Evaluating peformance when incorrect structure proposals produce very similar spectra to the correct structure
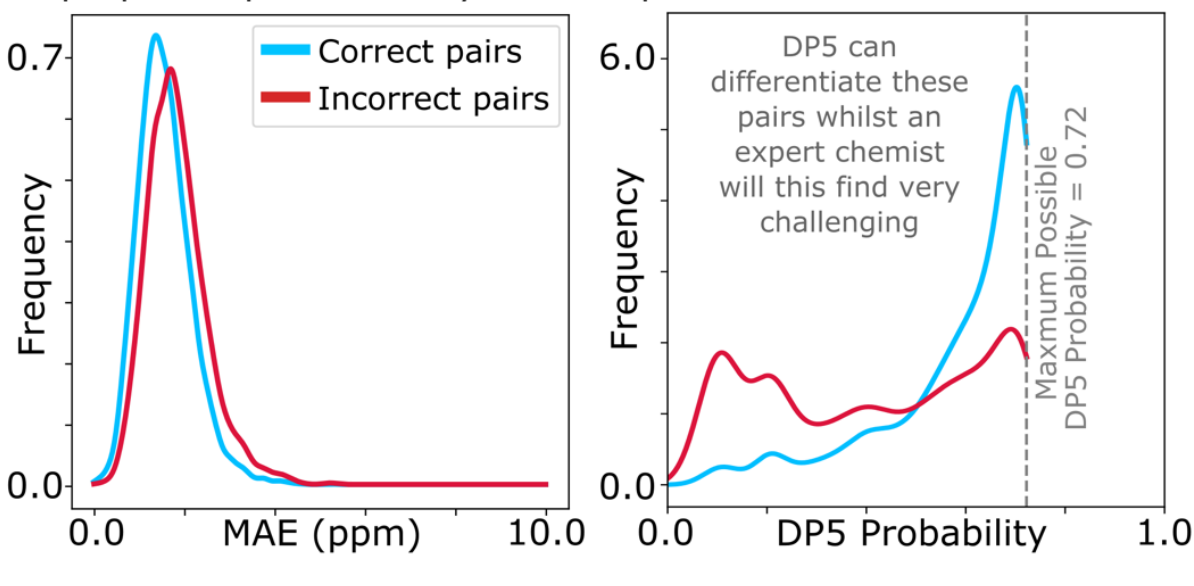

Figure 4. Schematic diagram of cross validation analysis used to evaluate the performance of DP5. A) The experimental spectra of the $5140(n)$ molecules from the NMRShiftDB training set are permuted to produce $n^{2}$ pairs of structures and experimental spectra. B) These pairs are separated into $n$ correct pairs and $n^{2}-n$ incorrect pairs, all incorrect pairs with max errors $<10$ ppm are considered in case 1 . C) In case 2 the incorrect pairs are assigned sampling weights to force their MAE distribution to approximate that of the correct pairs, this leads to an expected number of $\sim 5330$ incorrect combinations. All DP5 probabilities in this study are calculated using a leave-oneout scheme. (see supporting information section S3.2).

experienced chemists have acknowledged the difficulty of the challenge. The results of this study are shown in Figure 6.

\section{Discussion}

The results of the combinatorial cross-validation study are presented in Figure 4. In case 1, all incorrect pairs of structure and spectra with maximum errors $<10 \mathrm{ppm}$ are considered equally. This represents the situation where an experienced chemist should be able to accurately and reliably predict whether a chemically reasonable proposed structure is likely to be correct or incorrect based on the DFT-NMR prediction errors alone. There is very little overlap between the DP5 probability distributions for the correct and incorrect structure proposals, the modal DP5 probability for correct structures being the minimum possible value (see supporting information section 


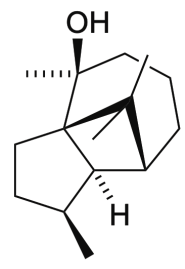

S1a

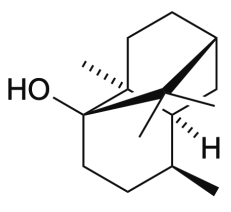

S1b<smiles>COc1cc2c(OC)c(C)c(=O)oc2cc1C</smiles>

S2a<smiles>COc1cc(OC)c2c(=O)cc(C)oc2c1C</smiles>

S2b<smiles>CC[C@@H]1CCC(=O)c2c(O)nc(C)c(OC)c21</smiles>

S3a<smiles>COc1c(C)[nH]c2c(c1=O)[C@@H](c1ccccc1)CCC2=O</smiles>

S3b<smiles>CC(=O)CC[C@@H]1C(C)(C)CC(=O)C[C@@]12CO2</smiles>

S4a<smiles>CC(=O)CC[C@H]1[C@H](C)CC2(C)CC(=O)C[C@@]12C</smiles>

S4b<smiles>CO[C@H]1[C@@H](C)CC[C@H]2C(C)=C3CCC(C)(C)[C@]21O3</smiles>

S6a

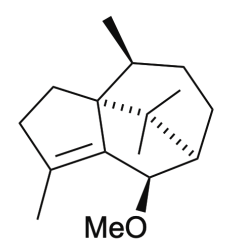

S6b

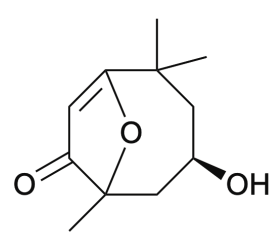

$\mathrm{S} 7 \mathrm{a}$<smiles></smiles>

$\mathrm{S} 5 \mathrm{a}$

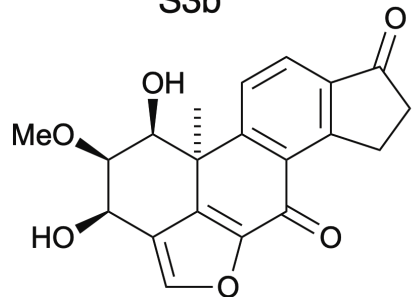

S5b<smiles>CC1(C)C[C@H](O)C[C@]2(C)OC(=O)C=C12</smiles>

S7b<smiles>O=C1OC2CC(=CC1O)[C@@H](O)C2</smiles>

S8a<smiles>O=C(O)C1=C[C@H](O)[C@H](O)[C@H](O)C1</smiles>
S8b<smiles>C#C/C=C\C[C@H]1O[C@@H]([C@H]2CC[C@@H](CC)O2)C[C@H]1Br</smiles>

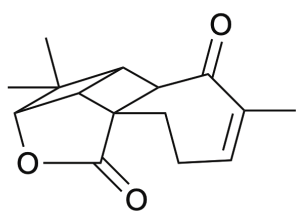

S10a<smiles>CC1=CCCC23C(=O)OC(C2C1=O)C3C(C)(C)C</smiles>

S10b<smiles>[SnH2]CCN=C(OCCC[SbH2])c1ccccc1</smiles>

S9a

S9b

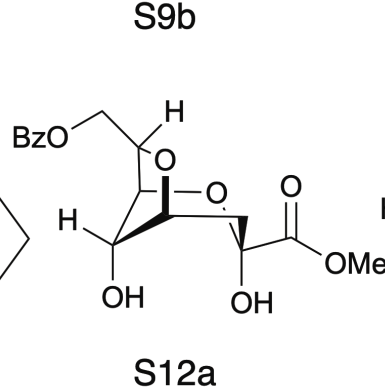<smiles>OC1CC2OC(C[18OH])C(C[C@H]1O)O2</smiles>

$\mathrm{S} 12 \mathrm{~b}$<smiles></smiles><smiles>COc1c(Br)cc(Br)cc1Oc1c(O)cc(Br)cc1Br</smiles>

Figure 5. Test set of real-world structure reassignment problems taken from chemical literature. In each example an incorrect structure was initially published (S\#a) which was later reassigned to the corresponding correct structure (S\#b)

S2.8). The incorrect structures display the opposite pattern, with the modal value at close to zero. This result highlights the DP5 probability's ability to differentiate reliably between correct and incorrect structures in these situations. Therefore, DP5 will perform as well as an experienced chemist when classifying structures based on DFT prediction errors alone. When paired this way, the correct and incorrect pairs show different MAE distributions, with the incorrect pairs displaying a larger modal MAE and a greater variance. The DP5 probability would be even more useful if it could reliably differentiate incorrect structure proposals following the same MAE distributions as the correct structures.

This is tested in Figure 4, case 2. The incorrect pairs are assigned weights to ensure that they follow the same MAE distribution as the correct pairs (see supporting information section S3.2).
This represents the radically more challenging situation where the correct and incorrect structure proposals are practically indistinguishable by their DFT prediction errors. In this situation an expert chemist would have significant difficulty deciding whether a proposed structure is correct or incorrect, and in some cases this may be impossible without collecting additional information. The results of this study are particularly exciting, as despite this test proving to be more demanding, the DP5 probability is still able to correctly differentiate many correct and incorrect structures. This is shown by the DP5 probability frequency distributions, with the correct pairs maintaining a strong peak at the maximum possible value and the incorrect pairs having significant density towards zero.

To further test the efficacy of DP5 analysis, the system was evaluated against thirteen challenging real-world examples, 
Figure 5. Not only are the correct and incorrect structures almost indistinguishable to an expert chemist, these structures have already been incorrectly assigned and revised in the literature. ${ }^{1,34-38}$ The results of this study are striking and are presented in Figure 6 . In all cases the DP5 probabilities of the incorrect structures are equal to or close to one, illustrating that DP5 can reliably pick out even the most subtle inconsistencies in molecular structures. These results illustrate the power of DP5 analysis: even for these extremely difficult examples, DP5 differentiates between the correct and incorrect structures There are three examples where the DP5 probabilities of the correct and incorrect structure are both equal to zero (S12, S2, $\mathrm{S} 13)$. This result is not surprising due to the complex and subtle rearrangements involved in these revisions. These results however do not show a weakness, but rather a distinct advantage of the DP5 probability over DP4. Being a single structure probability, DP5 is able to question the initial structure and the revision independently, if both structures are improbable DP5 can assign low probabilities to both. In these situations, when all the candidate structures are unlikely, DP4 probabilities must still sum to one and DP4 will typically randomly show overconfidence in one of the structures. This behaviour was clearly displayed when the analysis was repeated using DP4. Only in these three cases did DP4 assign any confidence to the incorrect structures and in two cases (S12 and S2) DP4 assigned the most confidence to the incorrect structures. The low DP5 probabilities for S8, S9 and S13, suggest DP4 may have assigned these structures correctly by chance. These results highlight the consequences of the underlying assumptions of the DP4 methodology. For DP4 probabilities to be reliable, the correct structure must be present in the list of candidates. When this is true, DP4 is more reliable and precise than DP5 as it is more sensitive to slight differences in NMR spectra and as more information is available within the calculation. This makes DP4 the perfect system when the correct structure is guaranteed to be in the list of proposals. However, in cases where none of the candidate structures may be correct, only the DP5 probability can reflect this and can be calculated to assess the reliability of the DP4 calculation.

These examples show how DP5 can serve as a valuable tool whenever a new molecule is made, increasing confidence when proposed structures are correct, highlighting cases where they are not and also playing a stern jury when an improbable but correct structure has been proposed. These results show how utilising DP5 analysis may have prevented these incorrectly assigned structures from being published.

A very interesting feature that these results illuminate is the value of the maximum possible DP5 probability. This value is dependent on many factors including, the dataset of atomic environments, the atomic representation chosen, and, most notably, the inherent uncertainty in the DFT NMR predictions. Using this state-of-the-art and highly-optimised set of conditions, DFT NMR predictions still have a MAE of $1.57 \mathrm{ppm}$. As a result, even if a proposed structure is correct, the DP5 probability has to take into account the possible variance in NMR predictions and reflect this uncertainty. Therefore, when using this set of DFT conditions, the user can never be more
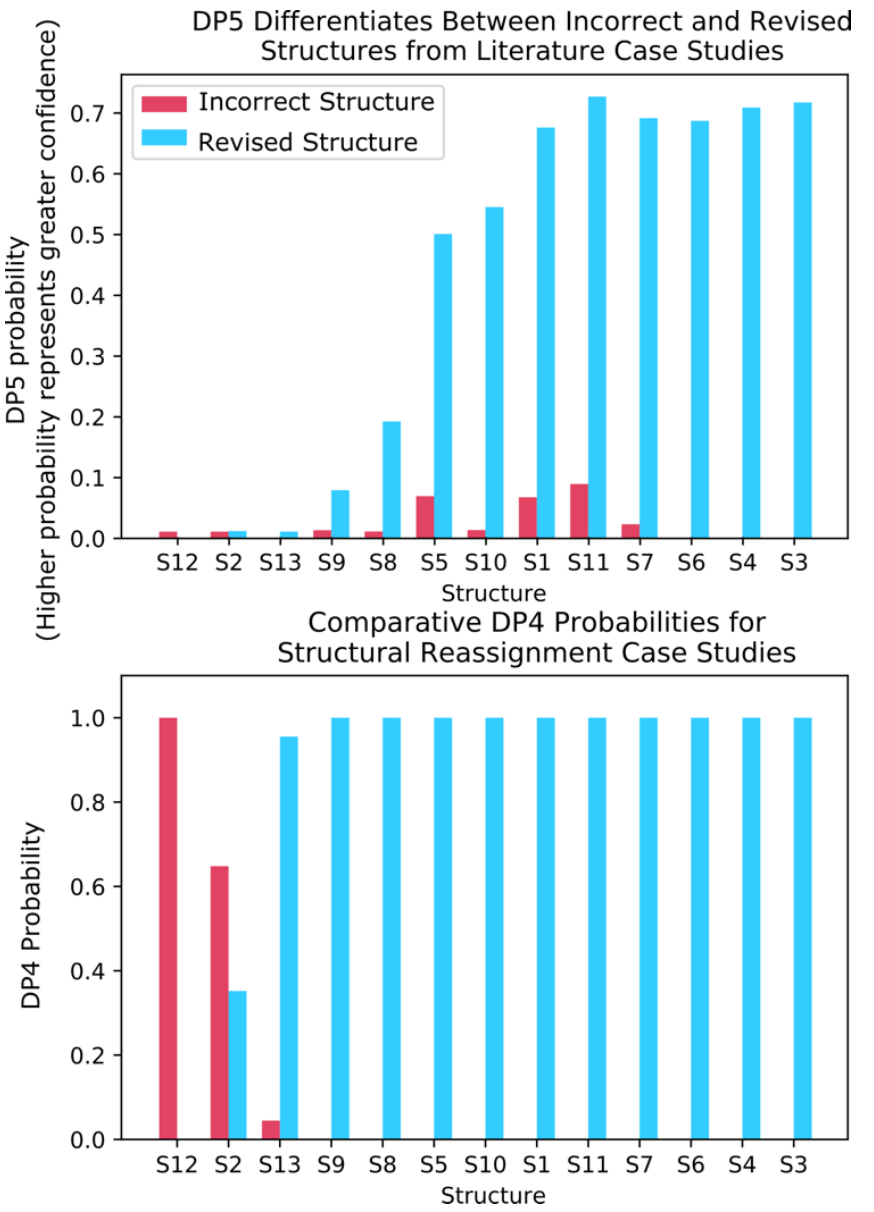

Figure 6. (top) DP5 probabilities calculated for the thirteen incorrectly published structures, and corresponding revised structures. DP5 assigns much greater confidence to the revised structures and also displays the three cases where both the initially proposed and revised structures are equally improbable. (bottom) DP4 probabilities calculated for the same thirteen examples. These results show how the DP5 probability can be used to test the reliability of a DP4 calculation, as only DP5 can discern if any of the structure proposals are likely to be correct.

than $72 \%$ confident that a structure is correct using one dimensional DFT NMR predictions alone. However, one can sometimes be $100 \%$ confident that a structure is incorrect. This value acts as a metric for assessing the accuracy of DFT NMR calculations and the reliability of the DP5 calculations. We expect that the use of even larger databases and even higher levels of theory will raise this limit. Equivalently, this can be interpreted as acknowledging that an incorrect structure could possibly produce a set of errors equally or more convincing than the correct structure, just as two molecules may produce similar experimental spectra. However, this is seldom a problem in organic chemistry as in most real-world applications, there are additional constraints on the potential structures that need to be considered. For example, in robot-controlled syntheses, the particular sequence of reactions is known limiting the potential products. In most cases, a DP5 probability of $73 \%$, combined with this additional data, will give the chemist much higher levels of certainty their structure is correct. In cases where 
multiple structures give high DP5 probabilities for the same spectra, this is a good indication of where DP4 can be applied in conjunction with DP5 to give even more accurate relative probabilities.

\section{Conclusions}

headings as appropriate. In conclusion, we have developed a new measure to quantify molecular structural uncertainty, the DP5 probability. This work represents a leap forward in quantification of structural uncertainty as, instead of a comparative dimensionless parameter, the probability of a structure being correct is quantified. This system was rigorously evaluated by a cross validation study and it was found that DP5 could perform as well as a human in classifying correct and incorrect structure proposals and in some cases could classify structures indistinguishable to a chemist. DP5 was evaluated against thirteen real-world examples of structures that were incorrectly published and subsequently revised in the literature. In all these challenging cases, DP5 expressed the maximum concern for the incorrect structures and was on average $41 \%$ more confident in the revised structures. The DP5 probability can be calculated fully automatically and so should find wide applications in uses cases such as high throughput reaction screening, automated chemical synthesis and drug discovery. In addition, DP5 may be run on a single molecule basis and the results explored utilizing the GUI, helping to guide the development of complex syntheses. This work also suggests how DP5 may be developed to help further to accelerate chemical discovery. Utilizing the DP5 alongside generative models and other machine learning methods to automatically guide structure determination being an attractive possibility. The DP5 system is available as open-source software at https://github.com/Goodman-lab/DP5

\section{Acknowledgments}

We thank EPSRC (A.H.) for financial support. We thank Dr Kristaps Ermanis for his advice and guidance on this project in addition to his continued work maintaining the DP4-Al repository.

\section{Conflicts of interest}

There are no conflicts to declare

\section{Notes and references}

1 K. C. Nicolaou and S. A. Snyder, Angew. Chemie Int. Ed., 2005, 44, 1012-1044.

2 K. C. Nicolaou, H. Zhang and A. Ortiz, Angew. Chemie Int. Ed., 2009, 48, 5642-5647.

3 K. C. Nicolaou, A. Ortiz and H. Zhang, Angew. Chem. Int. Ed. Engl., 2009, 48, 5648-52.

A. G. Kutateladze and T. Holt, J. Org. Chem, 2019,

84, 51 .

5
A. V. Buevich and M. E. Elyashberg, J. Nat. Prod., 2016, 79, 3105-3116.

P. Kessler and M. Godejohann, Magn. Reson. Chem., 2018, 56, 480-492.

J.-M. Nuzillard and B. Plainchont, Magn. Reson. Chem., 2018, 56, 458-468.

G. Barone, D. Duca, A. Silvestri, L. Gomez-Paloma, R. Riccio and G. Bifulco, Chem. - A Eur. J., 2002, 8, 3240 .

G. Barone, L. Gomez-Paloma, D. Duca, A. Silvestri, R. Riccio and G. Bifulco, Chem. - A Eur. J., 2002, 8, 3233.

G. Lauro and G. Bifulco, European J. Org. Chem., 2020, 2020, 3929-3941.

C. S. Kim, J. Oh and T. H. Lee, Arch. Pharm. Res., 2020, 43, 1114-1127.

S. G. Smith and J. M. Goodman, J. Am. Chem. Soc., 2010, 132, 12946-12959.

L. A. Maslovskaya, A. I. Savchenko, E. H. Krenske, S. Chow, T. Holt, V. A. Gordon, P. W. Reddell, C. J. Pierce, P. G. Parsons, G. M. Boyle, A. G. Kutateladze and C. M. Williams, Chem. - A Eur. J., 2020, 26, 11862-11867.

I. E. Ndukwe, X. Wang, N. Y. S. Lam, K. Ermanis, K. L. Alexander, M. J. Bertin, G. E. Martin, G. Muir, I. Paterson, R. Britton, J. M. Goodman, E. J. N. Helfrich, J. Piel, W. H. Gerwick and R. T. Williamson, Chem. Commun., 2020, 56, 7565-7568.

S. R. Lee, D. Lee, M. Park, J. C. Lee, H. J. Park, K. S. Kang, C. E. Kim, C. Beemelmanns and K. H. Kim, J. Nat. Prod., 2020, 83, 354-361.

J. S. An, J. Y. Lee, E. Kim, H. Ahn, Y. J. Jang, B. Shin, S. Hwang, J. Shin, Y. J. Yoon, S. K. Lee and D. C. Oh, J. Nat. Prod., 2020, 83, 2776-2784.

P. Yan, G. Li, C. Wang, J. Wu, Z. Sun, G. E. Martin, X. Wang, M. Reibarkh, J. Saurí and K. R. Gustafson, Org. Lett., 2019, 21, 7577-7581.

S. Kuhn and N. E. Schlörer, Magn. Reson. Chem., 2015, 53, 582-589.

O. A. von Lilienfeld, Int. J. Quantum Chem., 2013, 113, 1676-1689.

B. Huang and O. A. Von Lilienfeld, J. Chem. Phys., 2016, 145, 161102.

F. Brockherde, L. Vogt, L. Li, M. E. Tuckerman, K. Burke and K. R. Müller, Nat. Commun., 2017, 8, 1- 
10.

F. A. Faber, A. S. Christensen, B. Huang and O. A. Von Lilienfeld, J. Chem. Phys., 2018, 148, 241717.

A. S. Christensen, L. A. Bratholm, F. A. Faber and O. Anatole Von Lilienfeld, J. Chem. Phys., 2020, 152, 044107.

J. Townsend, C. P. Micucci, J. H. Hymel, V.

Maroulas and K. D. Vogiatzis, Nat. Commun., 2020, 11, 1-9.

K. Ermanis, K. E. B. Parkes, T. Agback and J. M. Goodman, Org. Biomol. Chem., 2016, 14, 39433949.

K. Ermanis, K. E. B. Parkes, T. Agback and J. M. Goodman, Org. Biomol. Chem., 2017, 15, 89989007.

A. Howarth, K. Ermanis and J. M. Goodman, Chem. Sci., 2020, 11, 4351-4359.

K. Ermanis, K. E. B. Parkes, T. Agback and J. M. Goodman, Org. Biomol. Chem., 2019, 17, 58865890 .

J. Li, J. K. Liu and W. X. Wang, J. Org. Chem., 2020, 85, 11350-11358.

M. Rupp, R. Ramakrishnan and O. A. Von Lilienfeld, J. Phys. Chem. Lett., 2015, 6, 3309-3313.

K. Hansen, F. Biegler, R. Ramakrishnan, W. Pronobis, O. Anatole Von Lilienfeld, K.-R. Mü and A. Tkatchenko, , DOI:10.1021/acs.jpclett.5b00831.

B. Huang and O. A. von Lilienfeld, Nat. Chem., 2017, 12, 945-951.

W. Gerrard, L. A. Bratholm, M. J. Packer, A. J. Mulholland, D. R. Glowacki and C. P. Butts, Chem. Sci., 2020, 11, 508-515. and D. Kim, J. Am. Chem. Soc., 2012, 134, 1178111790.

M. W. Lodewyk, C. Soldi, P. B. Jones, M. M. Olmstead, J. Rita, J. T. Shaw and D. J. Tantillo, J. Am. Chem. Soc, , DOI:10.1021/ja3089394.

B. Verbraeken, J. Hullaert, J. Van Guyse, K. Van Hecke, J. Winne and R. Hoogenboom, , DOI:10.1021/jacs.8b10918.

E. E. Podlesny and M. C. Kozlowski, J. Nat. Prod, , DOI:10.1021/np300141t.
2009 Schrodinger, LLC, New York, NY, .

T. A. Halgren, J. Comput. Chem., 1996, 17, 490-519.

T. A. Halgren, J. Comput. Chem., 1996, 17, 520-552.

T. A. Halgren, J. Comput. Chem., 1996, 17, 553-586.

T. A. Halgren and R. B. Nachbar, J. Comput. Chem., 1996, 17, 587-615.

T. A. Halgren, J. Comput. Chem., 1996, 17, 616-641.

T. A. Halgren, J. Comput. Chem., 1999, 20, 720-729.

I. Kolossv ry and W. C. Guida, J. Comput. Chem., 1999, 20, 1671-1684.

I. Kolossváry and W. C. Guida, J. Am. Chem. Soc., 1996, 118, 5011-5019.

and D. J. F. M. J. Frisch, G. W. Trucks, H. B.

Schlegel, G. E. Scuseria, M. A. Robb, J. R.

Cheeseman, G. Scalmani, V. Barone, B. Mennucci,

G. A. Petersson, H. Nakatsuji, M. Caricato, X. Li, H.

P. Hratchian, A. F. Izmaylov, J. Bloino, G. Zheng, J.

L. Sonnenberg, M. Had, 2009.

F. London, J. Phys. le Radium, 1937, 8, 397-409.

K. Wolinski, J. F. Hinton and P. Pulay, J. Am. Chem. Soc., 1990, 112, 8251-8260.

R. Ditchfield, J. Chem. Phys., 1972, 56, 5688-5691.

C. Adamo and V. Barone, J. Chem. Phys., 1998, 108, $664-675$.

W. J. Hehre, K. Ditchfield and J. A. Pople, J. Chem. Phys., 1972, 56, 2257-2261.

P. C. Hariharan and J. A. Pople, Theor. Chim. Acta, 1973, 28, 213-222.

F. Weigend and R. Ahlrichs, Phys. Chem. Chem. Phys., 2005, 7, 3297.

F. Weigend, Phys. Chem. Chem. Phys., 2006, 8, 1057.

E. Cancès, B. Mennucci and J. Tomasi, J. Chem. Phys., 1997, 107, 3032-3041.

58 A. D. Becke, Phys. Rev. A, 1988, 38, 3098-3100.

59 C. Lee, W. Yang and R. G. Parr, Phys. Rev. B, 1988, 37, 785-789.

60 Y. Zhao and D. G. Truhlar, Theor. Chem. Acc., 2008, 120, 215-241.

61 R. S. Guan, Yanfei, Paton, CASCADE, http://nova.chem.colostate.edu/cascade/, (accessed 8 March 2021). 
K. R. M. O. A. von L. A.S. Christensen, F. A. Faber,

B. Huang, L.A. Bratholm, A. Tkatchenko, GitHub

Repos. 